\title{
EQUIVALENT WIDTH OF H-ALPHA IN LATE-TYPE STARS
}

DONNA WEISTROP

Mount Wilson and Palomar Observatories

Carnegie Institution of Washington California Institute of Technology

Received August 8, 1967

The use of the equivalent width of $\mathrm{Ha}$ as a luminosity criterion for late-type stars is investigated. The method is found to be no more accurate than MK luminosity classifications. Two spectrograms are presented which indicate a change in the $\mathrm{H} a$ equivalent width in $22 \mathrm{Vul}$ over a three-year period.

Among absolute magnitude criteria for late-type stars more accurate than MK luminosity classifications, one of the most successful has been the Wilson-Bappu effect. The use of the Ca II emission line to determine absolute magnitudes has some disadvantages, however. Its location near $\lambda 3900$ is a major drawback in stars that have relatively weak fluxes in the ultraviolet. Measurement of the width of the emission against the central portion of a strong absorption line requires spectrograms of high spectral resolution and long exposure times. $\mathrm{H} \alpha$, on the other hand, is located in the red, where a large fraction of the flux of late-type stars is found, and, in addition, appears in absorption against this strong background. For the above, and other, reasons, the use of $\mathrm{H} \alpha$ as a luminosity criterion was investigated by Kraft, Preston, and Wolff (1964). It was found that there indeed exists a relation between the ultraviolet absolute magnitude, $M_{U}$, and the half-width of $\mathrm{H} \alpha$.

In order to use giants and supergiants for galactic structure studies, absolute magnitudes for stars of apparent magnitude 10 or 11 must be obtained. Since dispersions of 5 to $10 \AA / \mathrm{mm}$ are necessary to measure the half-width of $\mathrm{H} \alpha$, the exposure time needed is prohibitively long. Therefore, an investigation was initiated to determine whether the equivalent width of $\mathrm{H} \alpha$, which can be determined from plates of smaller dispersion, might also be useful as a luminosity criterion.

The spectrograms used in this study were taken with the 16 -inch camera of the Mount Wilson 60-inch Cassegrain spectrograph; the 
dispersion is approximately $30 \AA / \mathrm{mm}$, and Kodak IIa-E plates were used throughout. Values of the equivalent width of $\mathrm{H} \alpha, h$, were obtained from tracings made on the Caltech microphotometer.

The results of this study are given in Table I where $M_{U}$ is taken from Kraft et al. (1964). For those stars for which more than one spectrogram has been obtained, a mean value of $\log h$ is listed. The probable error of an equivalent width determined from one plate is $0.29 \AA$. Our results do not agree with those of Peat (1964), but considerable doubt has been cast on the individual equivalent widths he derives, owing to the width of his passband (Price 1966). It was impossible to compare the present results with those of Price (1966), owing to the small number of high-luminosity stars considered in his work.

Figure 1 illustrates the relationship between ultraviolet absolute magnitude and $\log h$. In spite of the scatter, a definite correlation between $M_{U}$ and $\log h$ is found. A least-squares solution gives for the straight line the relation

$$
\begin{aligned}
M_{U} & =2.38-17.45 \log h . \\
& \pm 0.61 \quad \pm 2.89 \text { (m.e.) }
\end{aligned}
$$

The probable error in $M_{U}$ determined from one observation is \pm 1.17 mag., considerably larger than the \pm 0.5 magnitude precision available from half-width measurements by Kraft $e t$ al. The size of the uncertainty indicates that the use of $\mathrm{H} \alpha$ equivalent widths as a luminosity criterion is no more accurate than MK luminosity classifications, and half-widths must be used to obtain improved absolute magnitudes, thus effectively eliminating this method from use in galactic structure studies.

There are indications from the data that the equivalent width of $\mathrm{H} \alpha$ changes in some stars. One of the most prominent cases is that of 22 Vulpeculae, which shows a change of about $30 \%$ in equivalent width over a three-year period. Plate I reproduces two spectrograms of $22 \mathrm{Vul}$, one taken in 1963, the other in 1966, which clearly illustrate the change in the hydrogen line. There is evidence that some other stars also show this effect, but the photometry is not accurate enough to provide definite proof. Further investigations should be undertaken to determine the extent of the $\mathrm{H} \alpha$ variations and the types of stars that display this phenomenon, since the possi- 


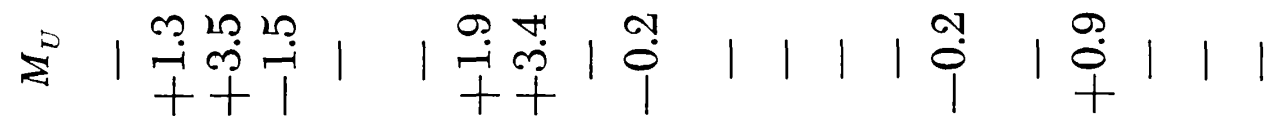

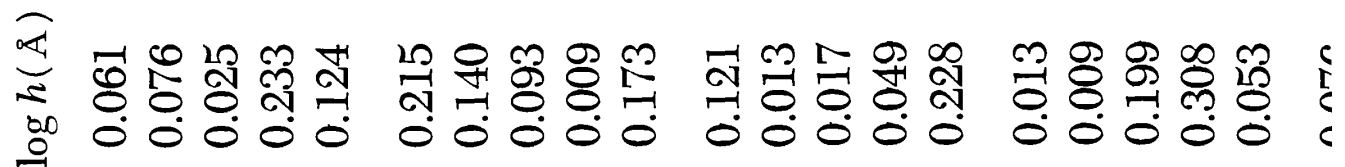

总 泀

迎

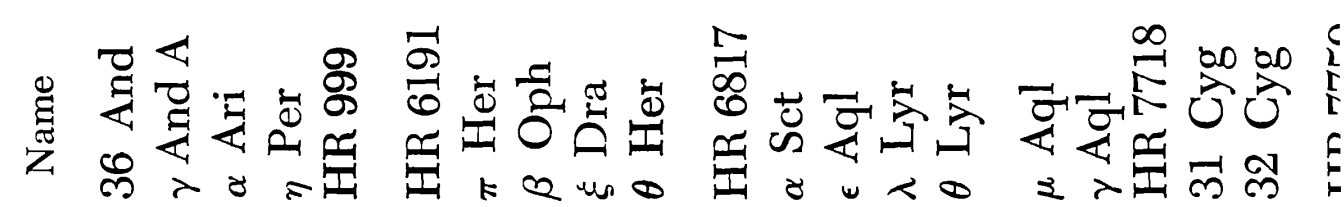

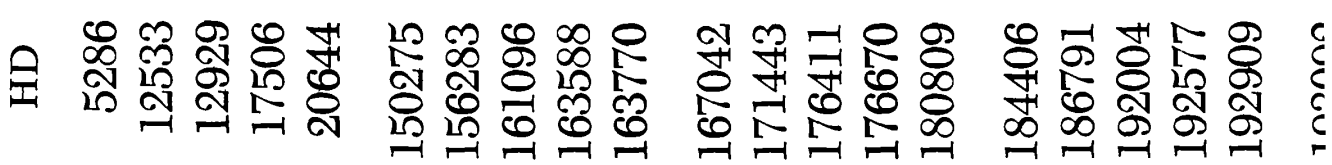

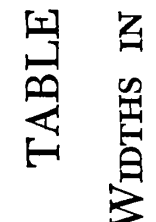

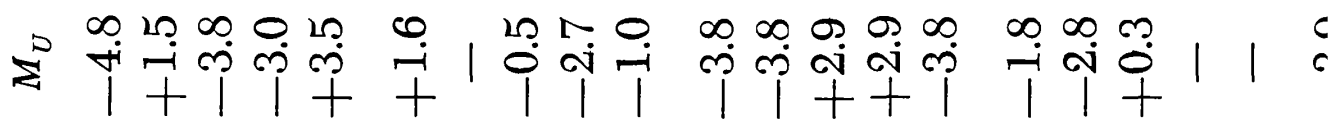

空

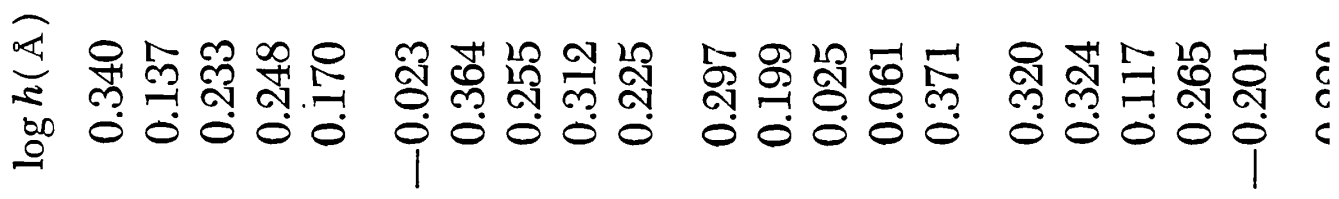

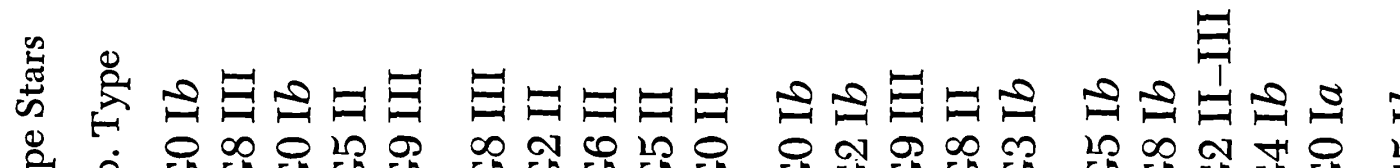
倠 क

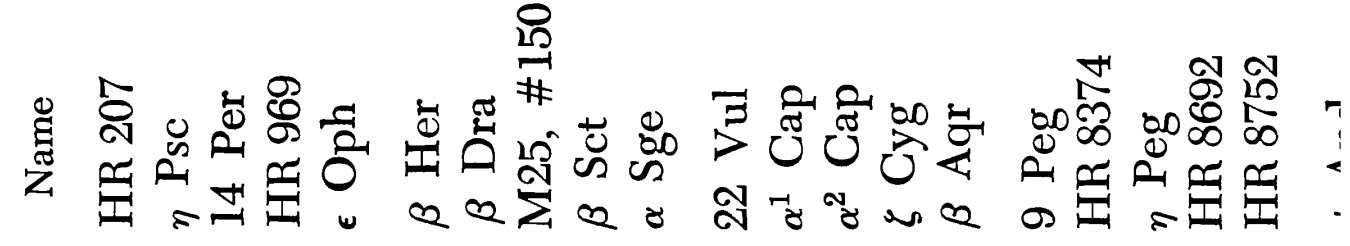

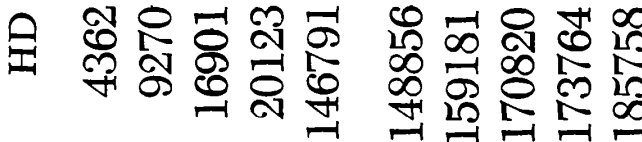

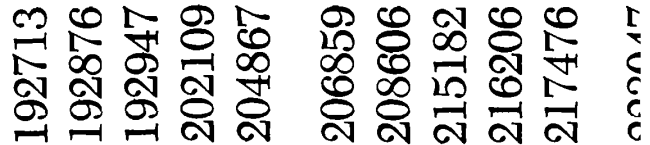




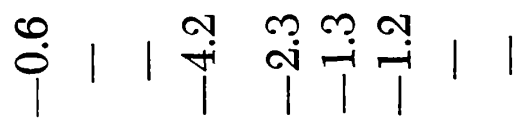

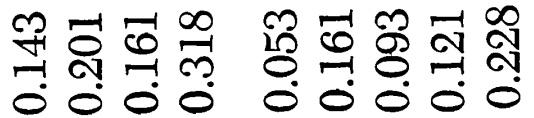

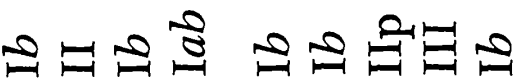

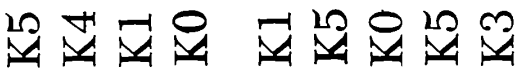

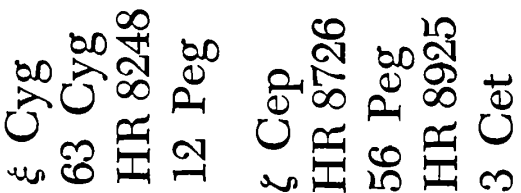

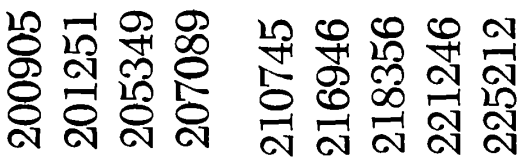
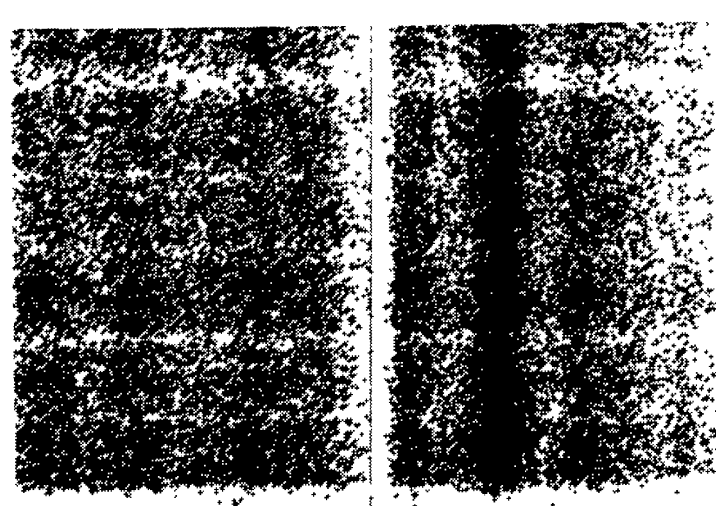

శْ
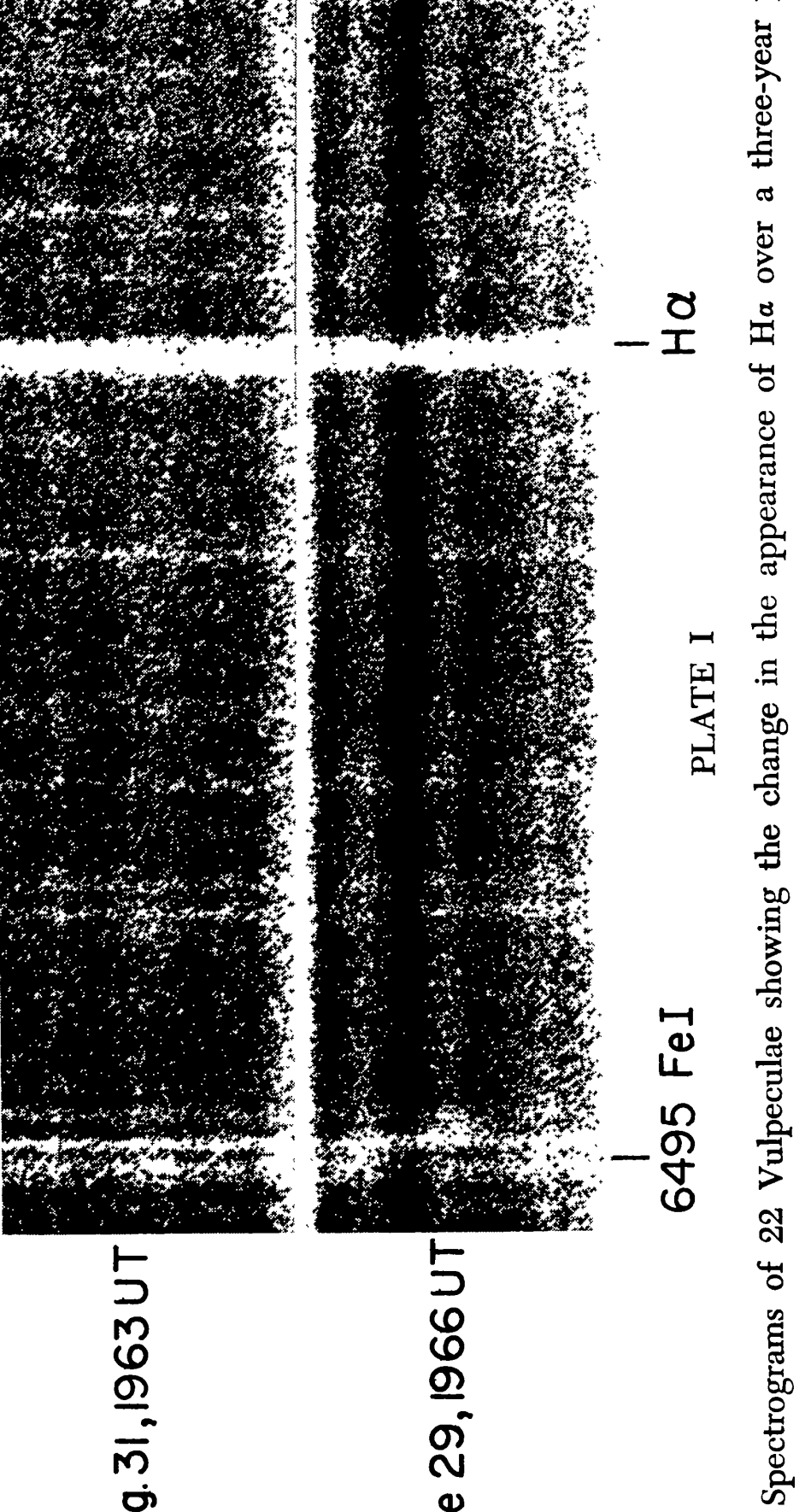

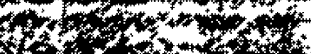

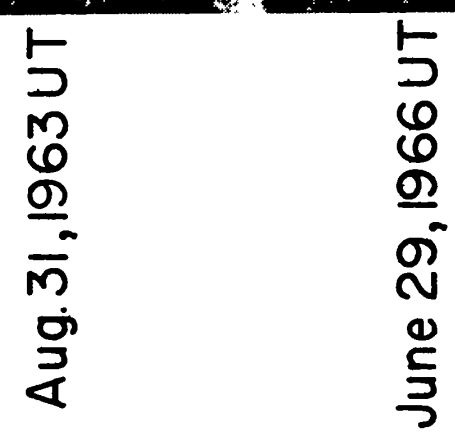

สิ

0

है: 


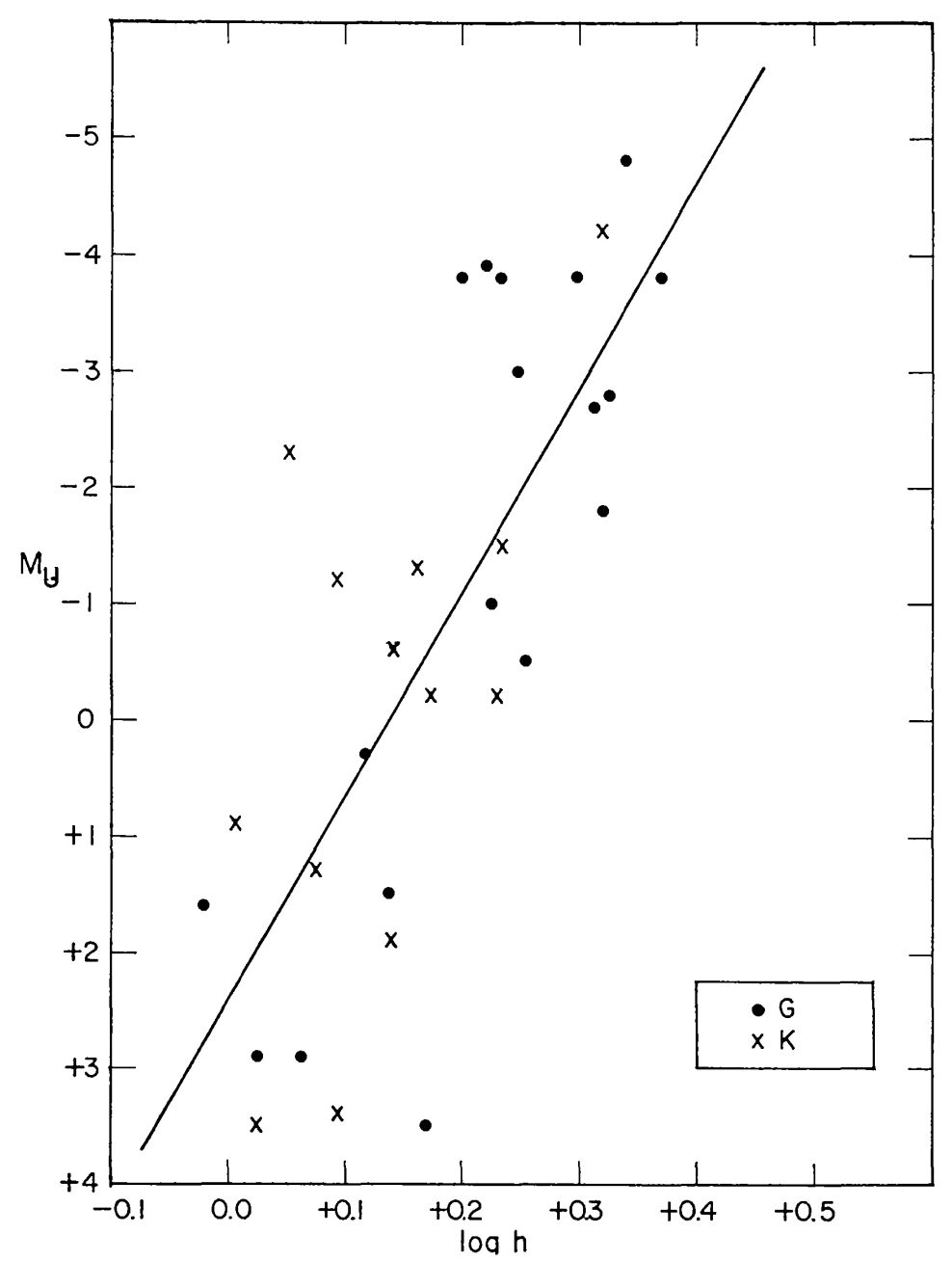

Fig. 1 - Correlation between $M_{U}$ and $\log h$ for the observed stars of known $M_{U}$. As noted, G-type stars are represented by dots and K-type stars by crosses.

bility of changes in the width of $\mathrm{H} \alpha$ casts considerable doubt on the use of $\mathrm{H} \alpha$ as a luminosity criterion.

I am indebted to Dr. R. P. Kraft for the use of the spectrograms taken by him in 1963 .

\section{REFERENCES}

Kraft, R. P., Preston, G. W., and Wolff, S. C. 1964, Ap. J. 140, 235.

Peat, D. W. 1964, M.N.R.A.S. 128, 435.

Price, M. J. 1966, M.N.R.A.S. 133, 449. 